\title{
Flexible, anti-damage, and non-contact sensing electronic skin implanted with MWCNT to block public pathogens contact infection
}

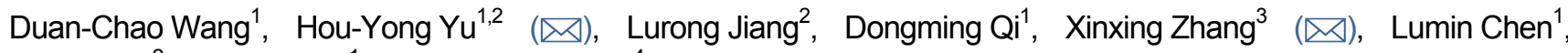 \\ Wentao $\mathrm{Lv}^{2}$, Weiqiang $\mathrm{Xu}^{1}$, and Kam Chiu Tam ${ }^{4}$ \\ ${ }^{1}$ National Engineering Lab for Textile Fiber Materials \& Processing Technology, College of Textile Science and Engineering, Zhejiang Sci-Tech \\ University, Hangzhou 310018, China \\ ${ }^{2}$ College of Information Science and Technology, Zhejiang Sci-Tech University, Hangzhou 310018, China \\ ${ }^{3}$ State Key Laboratory of Polymer Materials Engineering Polymer Research Institute of Sichuan University, Chengdu 610065, China \\ ${ }^{4}$ Department of Chemical Engineering, Waterloo Institute for Nanotechnology, University of Waterloo, 200 University Avenue West, Waterloo, \\ Ontario N2L 3G1, Canada
}

(C) Tsinghua University Press and Springer-Verlag GmbH Germany, part of Springer Nature 2021

Received: 27 May 2021 / Revised: 13 August 2021 / Accepted: 19 August 2021

\begin{abstract}
If a person comes into contact with pathogens on public facilities, there is a threat of contact (skin/wound) infections. More urgently, there are also reports about COVID-19 coronavirus contact infection, which once again reminds that contact infection is a very easily overlooked disease exposure route. Herein, we propose an innovative implantation strategy to fabricate a multiwalled carbon nanotube/polyvinyl alcohol (MWCNT/PVA, MCP) interpenetrating interface to achieve flexibility, anti-damage, and non-contact sensing electronic skin (E-skin). Interestingly, the MCP E-skin had a fascinating non-contact sensing function, which can respond to the finger approaching 0-20 mm through the spatial weak field. This non-contact sensing can be applied urgently to human-machine interactions in public facilities to block pathogen. The scratches of the fruit knife did not damage the MCP Eskin, and can resist chemical corrosion after hydrophobic treatment. In addition, the MCP E-skin was developed to real-time monitor the respiratory and cough for exercise detection and disease diagnosis. Notably, the MCP E-skin has great potential for emergency applications in times of infectious disease pandemics.
\end{abstract}

\section{KEYWORDS}

prevent contact infection, anti-damage sensors, non-contact sensing, respiratory sensing, human-machine interaction

\section{Introduction}

E-skin is an emerging material that can be used for wearable devices [1-4], health monitoring [5,6], soft electronics [7,8], human-machine interactions $[9,10]$, and intelligent robots [11-13]. More urgently, too little research proposed new physical properties and innovative structures to achieve non-contact sensing of E-skin. Applying the non-contact E-skin to the public human-machine interface can effectively block the public contact infection of various threatening pathogens. The threat of microbial pathogens to humans has always existed in human history. In the short 20 years of the 21st century, there have been three global epidemics, namely SARS, MERS, and the raging COVID-19. The World Health Organization (WHO) and related studies have confirmed that the SARS-CoV-2 virus not only spreads through droplets, but also occurs through direct contact with surfaces that have been used by infected persons $[14,15]$. If the hand touches a pathogen on a public facility and then touches the mucous membrane of the mouth or eyes, there will be a risk of infection. In daily life, pathogenic Escherichia coli can cause dysentery, gastroenteritis, urinary tract infections, and meningitis [16], and pathogenic Staphylococcus aureus can cause purulent inflammation and food poisoning [17], and various fungi can cause a variety of intractable skin disease [18]. More broadly, pathogens that cause chickenpox [19], hand-foot-mouth disease [20], measles [21], and other contact infect diseases are at risk of contact infection. Therefore, it is necessary to develop a noncontact electronic skin with low technical requirements, low cost, large-scale generation, and rapid promotion.

In previous E-skin research, most manufacturing strategies focused on gels [22-24] and multi-layer structure [25-28]. Because most flexible substrates are not suitable for lithography and existing E-skin strategies cannot be fabricated on uneven or irregular surfaces [29]. Only a few studies have focused on the antidamage (mechanical/chemical) and non-contact sensing performance of E-skin. For example, the anti-damage flexible sensing module use of liquid metal as microcapsules in elastomer can build a new sensing module to guarantee the continuous power supply of the soft electronic $[12,30]$. E-skin that can respond to finger motion without contact has more potential applications. Mao et al. prepared a multilayer structure gesture sensor based on tribo-electricity and electrostatic induction to control a phone without contact [31]. The preparation process 
includes chemical vapor deposition (CVD) synthesis of graphene, spin-on transfer graphene, magnetron sputtering indium-tin oxide (ITO) layer, laser etching ITO and other chemical etching process. It can be seen that it is not easy to fabricate a flexible non-contact sensing E-skin. Two-dimensional materials have been proven to have non-contact sensing capabilities, such as graphene [32], $\mathrm{MoS}_{2}$ [33], and $\mathrm{VS}_{2}$ [34], but they have very high requirements on the preparation process and substrate, and more difficult is the largearea or large-scale preparation, which limits their application in the civilian lives. Capacitive proximity sensors used to be applied in industry by capacitance affected by displacement, that is an interesting idea but they often need interdigital electrode and oscillation circuit [35]. So we should improve a low cost, universal, and suitable for large area manufacturing way to fabricate a noncontact E-skin for widely using in the human-machine interactions of public facilities.

Herein, we present a universal, rapid prototyping, and cheap implanting way to fabricate one-piece multi-walled carbon nanotube/polyvinyl alcohol (MWCNT/PVA, MCP) E-skin. MWCNT has the characteristics of light weight, ballistic conductivity, electrochemical induction, high compressive strength, and resilience [36,37]. Biocompatibility, film-forming properties, and gelation of PVA make it a popular electronic skin substrate [38-40]. Implanted MWCNT into the surface of PVA film results in flexible and anti-damage MCP E-skin with noncontact sensing. The sensitive sensing capabilities of MWCNT enabled the MCP E-skin to detect the proximity signal of finger from 0-20 mm. This urgently needed non-contact sensing MCP Eskin can be applied to non-contact human-machine interactions in public facilities to effectively block the contact infection of pathogens. The flexibility and moderate anti-damage (mechanical damage/chemical corrosion) make the MCP E-skin not easy to damage during transport and daily use. In addition, the MCP Eskin can sense the breathing and cough of the human body, and can distinguish the respiratory condition and the severity of the cough, which can be applied to the diagnosis and treatment of pneumonia. In short, it is a universal, rapid prototyping, lightness, stability, multi-function, and low price E-skin fabricate strategy. A unique MCP E-skin has been fabricated that was simultaneously flexible, non-contact sensing, resistant to mechanical damage, chemically resistant, and tactile mapping performance. In contrast to the graphene-based non-contact E-skin described above, the MCP E-skin has low technical requirements and is suitable for large-scale manufacturing, and thus can be potentially applied in non-contact public human-machine interactions, smart robots, and tactile sensors.

\section{Results and discussion}

\subsection{Fabrication of MCP E-skin}

It's a challenge to fabricate flexible sensing modules on polymer interfaces since mostly conductive materials have poor combine ability with macromolecule substrate [29]. Different from others expensive and energy intensive researches [41], we found that the PVA interfaces would be able to effectively composite with MWCNT by a snipped fountain pen, as shown in Figs. 1(a) and 1(b). The nib of the pen was cut by pliers to increase the sharpness and friction (Fig. S1 in the Electronic Supplementary Material (ESM)). The snipped fountain pen tip can not only puncture the polyvinyl alcohol (PVA) film surface but also implant the MWCNT, meanwhile, $\mathrm{H}_{2} \mathrm{O}$ dispersion dissolves the PVA film surface. The MWCNT cannot be adhered to the PVA film stably without that process. As shown in Fig. 1(a), the snipped fountain pen tip forcefully punctured the smooth surface of the PVA and left a shallow groove, then dipped the MWCNT solution to implant them into the groove. Figure 1(b) shows a schematic diagram of the mechanism of this process. In the process of forming grooves and transporting MWCNT, the jagged fault of fountain pen tip cut through the dense and smooth molecular chain layer on the PVA surface (this dense molecular chain seems to be the barrier between the conductive substance and the PVA film), and the groove became a reaction vessel for the mutual penetration of MWCNT solution and PVA molecular chains. The $\mathrm{H}_{2} \mathrm{O}$ in the MWCNT solution participated in the dissolution and destruction of the molecular chains of the PVA surface layer. The dissolved PVA molecules on the surface layer had a certain viscosity and adhered to the MWCNT. The structure of mechanical interlocking and the mechanism of adhesion are shown in Fig. 1(c), PVA penetrated into the gaps and bumps of
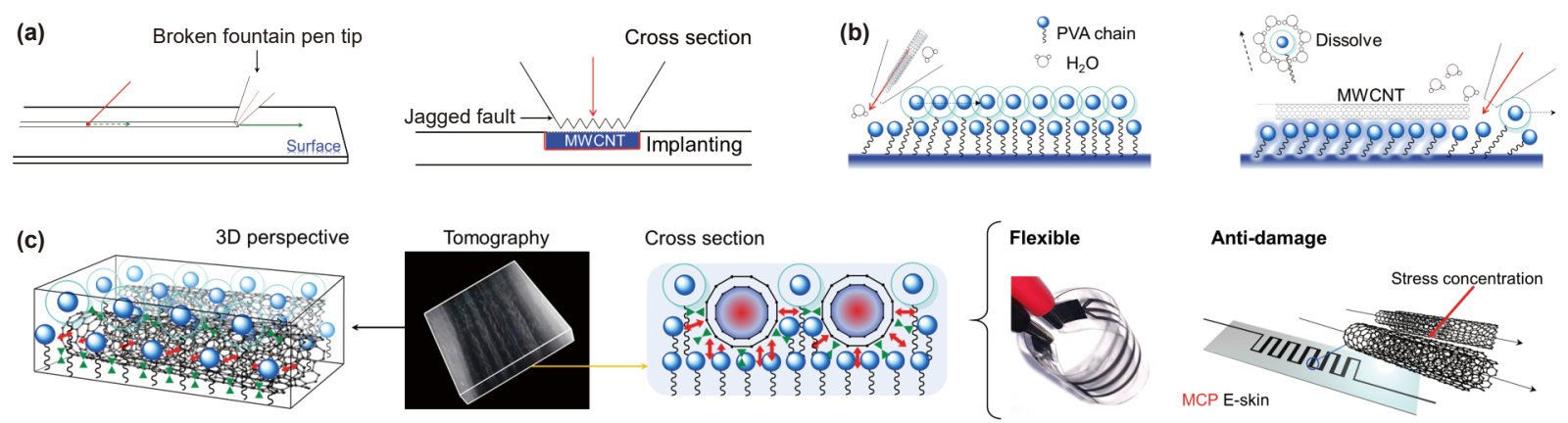

(d)
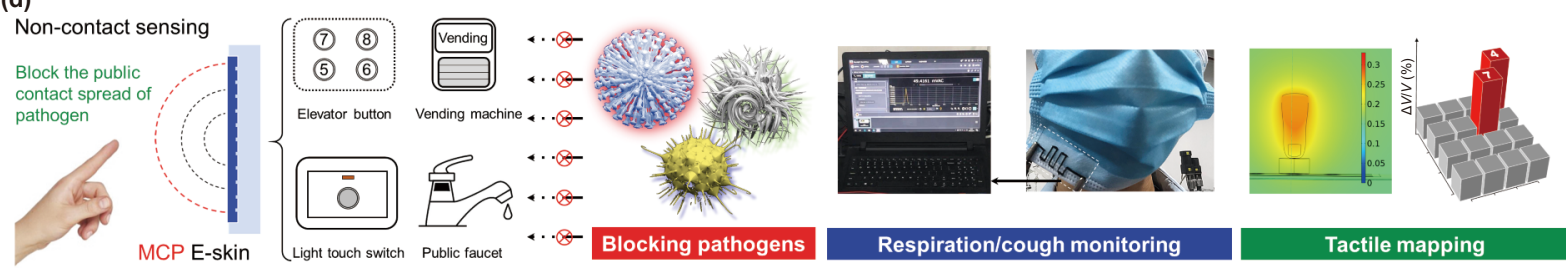

Figure 1 Schematic diagram of fabricated MCP E-skin and its unique non-contact sensing performance. (a) The process of implanting MWCNT in the surface of the PVA film. (b) Schematic diagrams of MCP E-skin fabrication. (c) Confocal laser tomography $(4,500 \mu \mathrm{m} \times 4,500 \mu \mathrm{m} \times 400 \mu \mathrm{m})$ discovered PVA and MWCNT permeated each other to form a mechanical interlocking structure (green represents van der Waals force and red represents hydrogen bond), which structure bought the flexible, anti-damage performance to MCP E-skin. (d) The non-contact sensing capability of MCP E-skin allows citizens to use a variety of public non-contact human-machine interfaces, which reduces the probability of contacting pathogens in the public environment. In addition, MCP E-skin can also monitor human breathing/coughing and achieve tactile mapping. 
MWCNT, and formed a meshing force in the interface area after curing. The main source of bonding force is the intermolecular force when the two materials contact, including van der Waals force and hydrogen bonding force [42]. This process would be repeated 3-5 times, and MWCNT would be stacked and connected to each other to form an electron transmission channel (Fig. S2 in the ESM). In order to observe the combined state of MWCNT and PVA, the shallow surface (400 microns depth) of MCP E-skin was scanned with a laser scanning confocal microscope, as shown in Fig. 1(c) and Fig. S3 in the ESM. The 3D tomography showed that MWCNT and PVA formed an interpenetrating structure. They were interspersed and oriented like a mechanical interlocking structure, which helped the two to be firmly meshed after curing [42]. The electron transmission channel formed by MWCNT was only a few microns deep and can sensitively sense various signals in fellow test. On the other hand, this interesting mechanical interlocking structure brought excellent flexibility, stability, and anti-damage performance to MCP E-skin. Figure S2(c) in the ESM shows the analogy between real skin and MCP E-skin. MCP E-skin not only had the stratum corneum like real skin to protect itself, but also surprisingly, it also had the non-contact sensing ability like body hair. The expected non-contact sensing capability has been successfully fabricated, and the MCP E-skin of this study would be applied to block the contact infection of pathogens. In public places, people who carry or have been infected with pathogens have a high probability of transmitting the virus to public facilities, such as elevator buttons, light touch switches, vending machines, and faucets in Fig. 1(d). Replacing these switching mechanisms with MCP E-skin will effectively block healthy citizens from being infected by the virus, thereby blocking their infection. Therefore, the MCP E-skin with non-contact sensing capability has great hopes to be used in public facilities to cut off the contact infection of pathogens.

This research proved a novel and general method of preparing flexible non-contact E-skin. In recent studies, ion skin gel structures and multilayer structures were more popular for E-skin materials (Fig. 2(a)). However, the E-skin of these two structures does not have deformation resistance stability and damage resistance. The strategy of implanted MWCNT in this study does not need to incorporate ions, nor does it require a layer to layer (a)

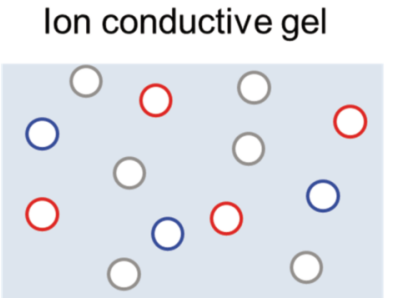

Multi-layer structure

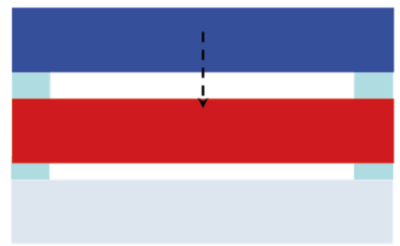

* This work MCP E-skin

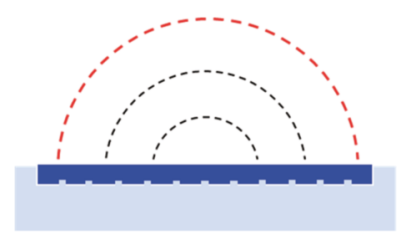

(b)

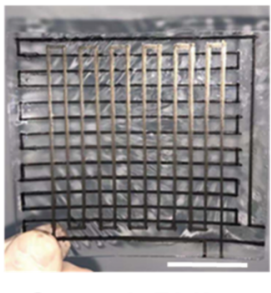

Smooth PVA

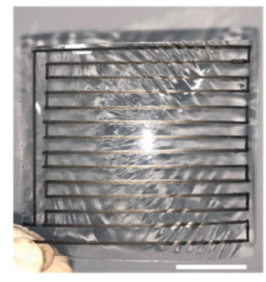

Crumpled PVA

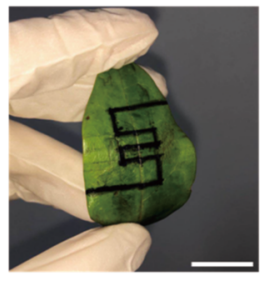

Holly leave

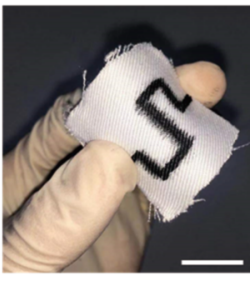

Cotton cloth

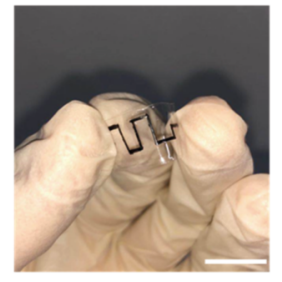

Cellulose paper

(c)

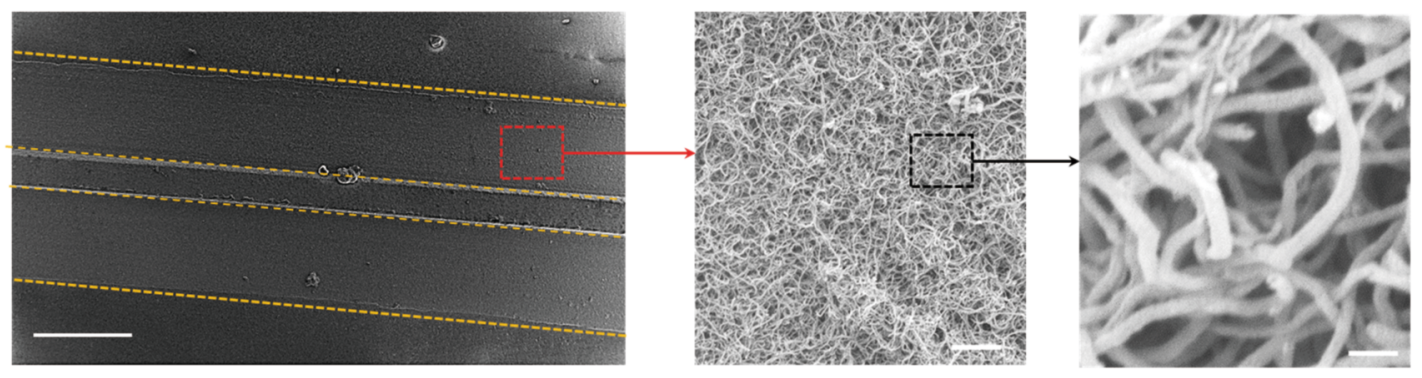

(d)

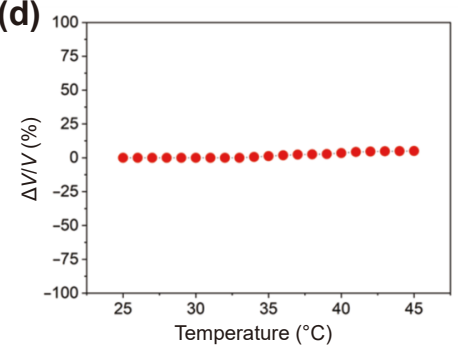

(e)

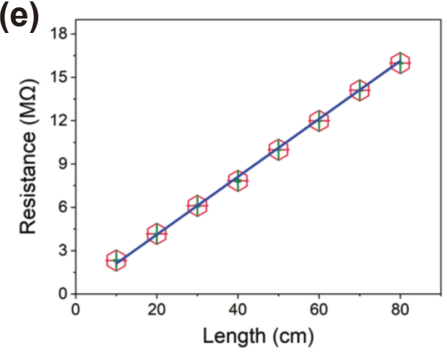

(f)

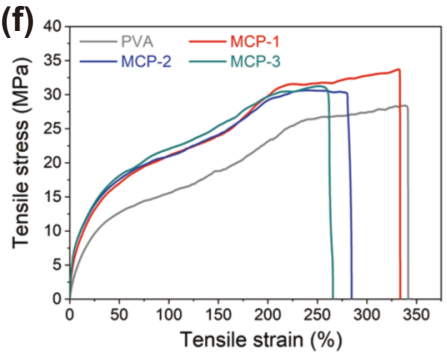

Figure 2 Versatile and regulated MCP E-skin implanted with MWCNT. (a) A schematic diagram of different structural strategies of this study and the current popular E-skin. (b) Photos of different flexible MCP E-skins, included smooth PVA, crumpled PVA, holly leaves, cotton cloth, and cellulose membrane, scale bar: 5 $\mathrm{cm}$. (c) FE-SEM image of an MCP E-skin sensing module (scale bar: $100 \mu \mathrm{m}$ ) and MWCNT in the MCP E-skin sensing module (scale bar: $1 \mu \mathrm{m}$ and $200 \mathrm{~nm}$ ). (d) The curve of resistance changing with temperature. (e) Effect of length on sensing module resistance. (f) Stress-strain curves of pure PVC film and MCP, the numbers represent the number of circuits on the MCP. 
precise alignment technologies. Figure 2(b) shows that the implanting strategy can adapt to different substrates, included smooth PVA, crumpled PVA, holly leaves, cotton cloth, and cellulose membrane, suggesting a good versatility. Field emission scanning electron microscopy (FE-SEM) images (Fig. 2(c)) show that the single MWCNT sensing module had a regular and continuous structure. Further magnification found that the distribution of MWCNT was unexpectedly uniform and continuous. These ideal morphologies implied that the MWCNT sensing module prepared by this rapid prototyping implanting method was not inferior to the other researches. Ultraviolet-visible (UV-vis) spectrum (Fig. S3(a) in the ESM)) analysis confirmed that the dispersion of MWCNT in the solution was good, and the samples with three concentrations all showed the characteristic peak of MWCNT at $202 \mathrm{~nm}$, this is due to the $\pi$ $\rightarrow \pi^{*}$ transition caused by the resonance absorption of energy by $\pi$ free electrons on the carbon nanotube surface [43]. The function relationship between the parameters and resistance of MCP sensing module through actual measurement and theoretical model is shown in Fig. 2 and Fig. S4 in the ESM. Among them, there was a standard linear relationship between the length variable ( $L e$ ) and the resistance, and $R^{2}$ reached 0.998 (Fig. 2(e)). That was consistent with Kaisor's Heterogeneous Fibrillar Model $[44,45]$. The concentration $(C)$ of MWCNT, the width $(W)$ of sensing module, and the layer $(L)$ of sensing module discussions are in the ESM. The high matching between the measured data and Kaisor's model implied the uniformity, continuity, and adjustability of the implanting sensing module. Figure 2(f) shows the stress-strain curves of PVA film and MCP. Obviously, the MWCNT circuit improved the tensile strength, but reduced the toughness, and that became obvious as the number of MWCNT circuits increased. The Young's modulus of PVA, MCP-1, MCP2, and MCP-3 were 19.21, 31.81, 29.37, and $29.16 \mathrm{MPa}$, respectively. This was because MWCNT/PVA formed mechanical interlocking interfaces, and the high strength and low toughness of MWCNT were combined with the original characteristics of PVA film. After the number of circuits was increased to 3, the Young's modulus was slightly decreased, which may be caused by the increased surface dissolution of PVA, but it was still much larger than that of pure PVA. Even if the MCP was stretched to stress whitening, the combination of the two was stable without any signs of detachment or breakage (Fig. S5 in the ESM). This mechanically proves the good combination of MWCNT and PVA. Compared with E-skin with hydrogel structure and multilayer structure, such a one-piece, universal, and rapid prototyping MCP E-skin with high regularity and high strength is advanced [22-28].

\subsection{Flexible and anti-damage performance of MCP E-} skin

Novel implanted structures bring exciting characteristics to MCP E-skin, such as, non-contact sensing without direct contact, antidamage, and resistance stability after large angle deformation. It is necessary to keep the resistance unchanged when the flexible sensing module is deformed such as bending, which can ensure the stability of the signal transmission of the intelligent system [46]. We chose the best sample preparation parameters of 100 $\mathrm{mg} / 20 \mathrm{~mL}$ (MWCNT/liquid), $2 \mathrm{~cm}$ width, and 3 layers. Figures $3(\mathrm{a})$ and $3(\mathrm{~b})$ show that the resistance change ratio was very small of the implanted MWCNT sensing module after bending $\theta=360^{\circ}$ or $720^{\circ}$, with changes of $2.8 \%$ and $6.8 \%$. The photo of flat MCP E-skin was shown in Fig. S6(a) in the ESM and the bending angle model was shown in Fig. S6(b) in the ESM. During bending, the circuit of the MCP E-skin was close to the neutral layer, which made it neither compressed nor stretched, so its resistance remained almost unchanged (Fig. S6(c) in the ESM). This can ensure that the flexible sensing module system maintains the stability after deformation, such as the deformation of the MCP E-skin when installed in public facilities will not affect its essential performance. Figure 3(c) shows the optical microscope and FE-SEM images of MCP E-skin cut by a fruit knife 4 times. The damage was found to be a white dent, and the MWCNT on the MCP E-skin showed a partial collapse but did not break. After three cuts, the resistance increased to about 78\% (Movie ESM1) and did not invalidate the sensing performance (Figs. 3(d) and 3(e)). Figure 1(e) found that the MWCNT sensing module and PVA formed a mechanical interlock structure, which was conducive to the stable fit of the two. The excellent nanomechanical properties of MWCNT, including extraordinary specific stiffness, high compressive strength and resilience, played a key role in resisting damage (Fig. 3(d)). On the other hand, the surface of the PVA film prepared by natural volatilization was relatively compact. Cutting will only "squeeze" the implanted sensing module without completely destroying it. The MCP Eskin resistance can be restored after implanted MWCNT again, as shown in Fig. S7 in the ESM. We used a steel ruler to scratch it to simulate possible bumps. The resistance of the MCP changed very little, and the resistance after four scratches only increased by $4.8 \%$ (Fig. 3(f)). MCP E-skin was coated with a thin layer of silicone oil to avoid water or chemical attack. What is exciting is that this protective layer not only effectively resisted the damage of water, acid, and lye (Figs. 3(g) and 3(h)), but also did not hinder noncontact sensing. The water contact angle and water washing test implied the good chemical resistance of MCP E-skin, and the noncontact sensing performance was not damaged in water washing and coating (Movie ESM2). Compared with the E-skin that requires comfortable working environments in previous studies $[29,31-34]$, the resistance of mechanical and chemical damage exhibited by MCP was very beneficial to the long-term stability of human-machine interface applications, which makes it easier to move from the lab to real-life use.

\subsection{The non-contact sensing performance of MCP E-skin and its application}

The non-contact sensing capability of MCP E-skin is delightful, because most flexible tactile sensors are operated by directly touching the sensing unit (MCP E-skin can also achieve this, Fig. S8 in the ESM). What is exciting is that the MCP E-skin in this study can sense the finger without contact. The physical model on which this phenomenon is based is the principle of plate capacitance, please see Fig. S9 in the ESM and its description for the specific model and principle. Figure 4(a) and Fig. S10 in the ESM show the non-contact sensing scene and finite element simulation scene of the MCP E-skin in the range of 0-20 mm. The super-sensitive sensing ability of the implanted MWCNT gave the MCP E-skin to capture the signal of the finger approach in advance and caused the resistance (voltage) to change, as shown in Fig. 4(b) and Movie ESM2. If this unique ability is applied to the human-machine touch interface of public facilities, it can completely block the contact infection of pathogens, which is undoubtedly an advanced emergency application. The noncontact sensing range was $0-20 \mathrm{~mm}$, which not only prevents contact infection but also prevents mistouch. Figure 4(c) shows a simulation scene of switching lights without touching them. Through simple circuit logic and connection, the resistance threshold was triggered when the finger approached the MCP Eskin for the first time, and the circuit automatically turned on the light-emitting diode (LED) light; when the finger approached again, the LED light off instruction was triggered. The finger did not have any contact with the switch during the entire operation. 
(a)

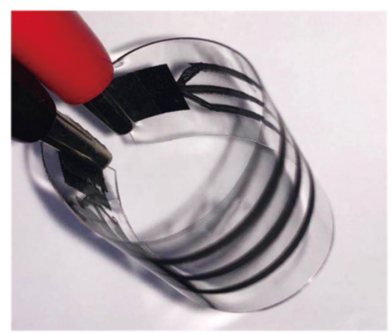

Bending angle $\theta=360^{\circ}$

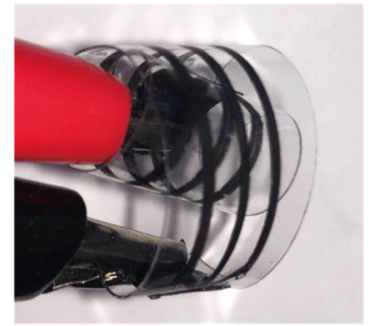

$\theta=720^{\circ}$

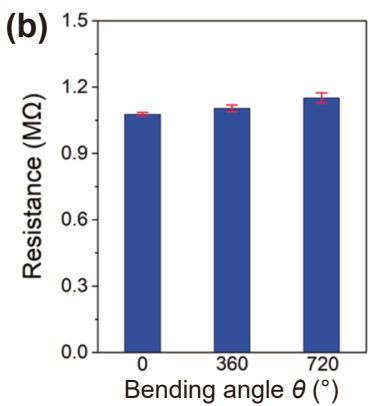

(c)

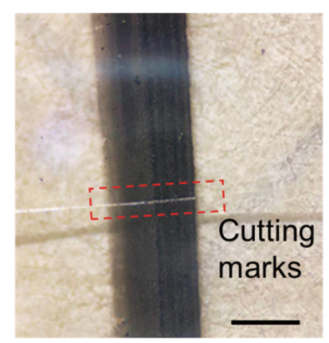

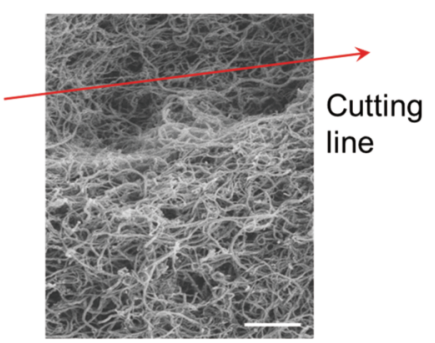

Cutting

e (d)

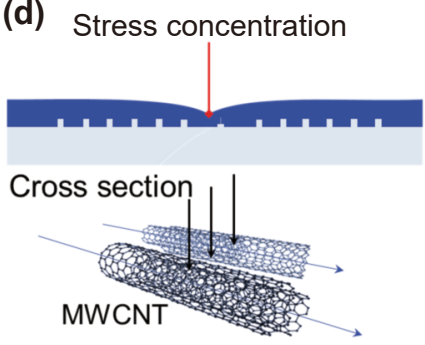

(e)

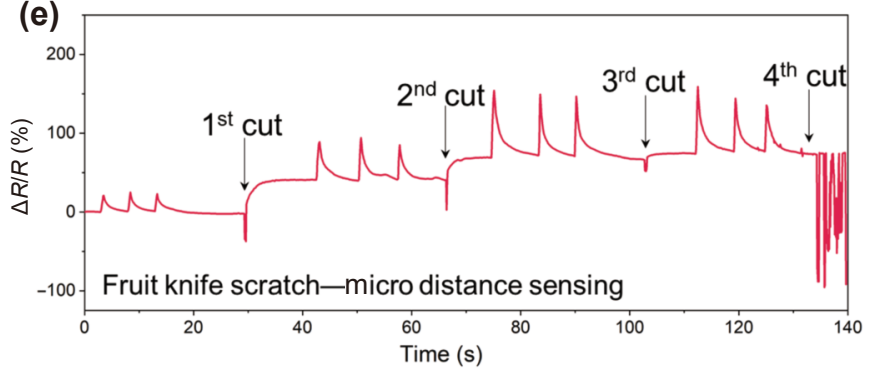

(f)

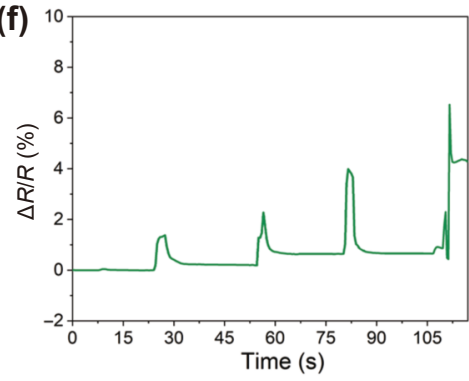

(g)
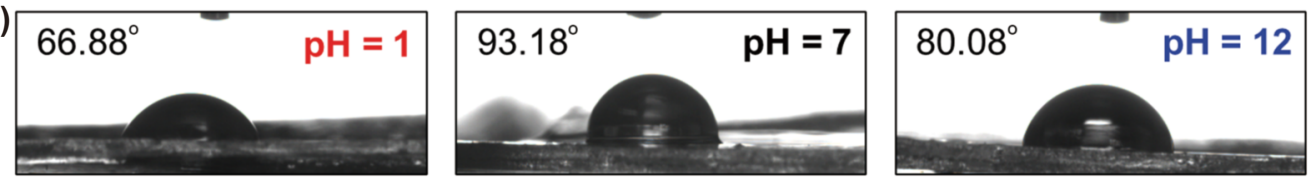

(h)
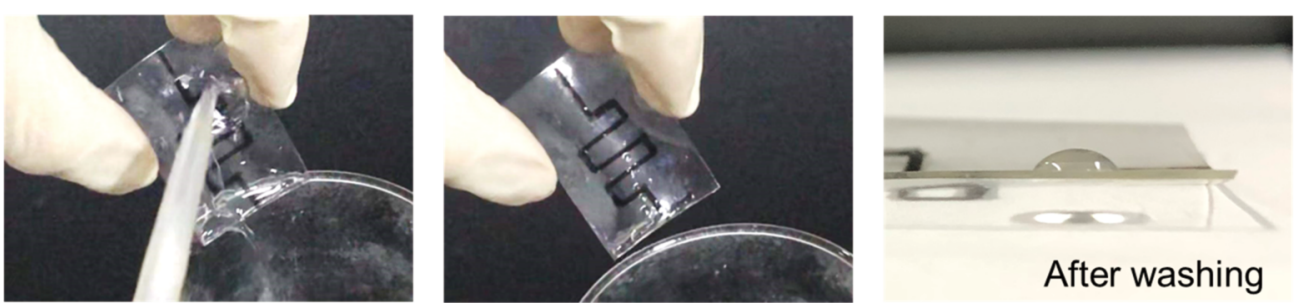

After washing

Figure 3 Unique performances of MCP E-skin. (a) MCP E-skin flexibility test, curled it $\theta=360^{\circ}$ or $720^{\circ}$, respectively. (b) Resistance change after curled. (c) Microscope and FE-SEM images of MCP E-skin after fruit knife 4 times cut. Scale bar: $1 \mathrm{~mm}, 1 \mu \mathrm{m}$. (d) Schematic diagram of the possible mechanism of anti-damage performance. (e) Resistance change during fruit knife 4 times cut and non-contact sensing. (f) The resistance changes of MCP E-skin after steel ruler four times cut. (g) $1 \mathrm{M} \mathrm{H}_{2} \mathrm{SO}_{4}$, water, and $1 \mathrm{M} \mathrm{NaOH}$ contact angle of MCP E-skin (silicone oil coating). (h) The photo of the MCP E-skin (silicone oil coating) washed with water and the photo of the contact angle of the large volume of water after washing.

The cycle test showed that its non-contact sensing ability was stable, as shown in Fig.4(d). This once again verified the practicality of the MCP E-skin, and its large-scale application to the human-machine interface of public facilities is very promising to block the contact infection of pathogens.

Another interesting application is the monitoring of vital signs of the human respiratory system by MCP E-skin, including respiratory status and cough frequency. As shown in Fig. 4(e), the curve of normal breathing was soothing and the frequency was low, and the curve of simulated dyspnea was rapid and the response amplitude was low. This is because the single exhaled gas was less and the frequency was high. The tester attached the MCP to the mask and performed a 20 min breathing monitoring of daily activities, and the data is shown in Fig. 4(f). The human body movement of sitting or walking affected breathing rate and intensity, which caused the original breathing sensor signal to be untidy. However, the performance of MCP was not inferior to the commercial humidity/respiration sensor and cutting-edge respiratory sensing materials $[47,48]$, and these original signals can be processed by back-end processing such as rectification and amplification (Fig. 6 below simply shows this process) [8]. MCP Eskin can monitor the intensity and frequency of human breathing to help people better understand their motor function. Figure 4(g) shows the breathing change process of an adult male after running for one kilometer on a treadmill. Interestingly, the whole process of "rapid breathing-respiratory rate slowing-breathing back to normal" was fully and clearly recorded by MCP. In addition, MCP can also screen breath/cough and monitor the severity of cough. 
(a)

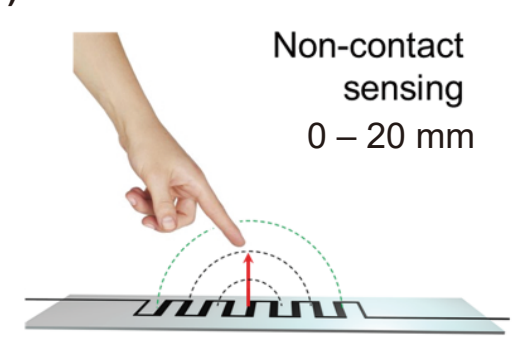

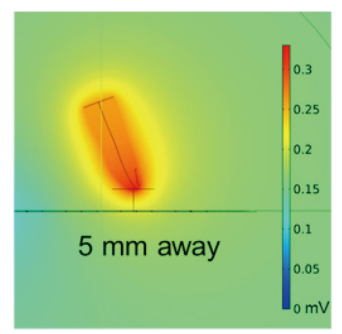

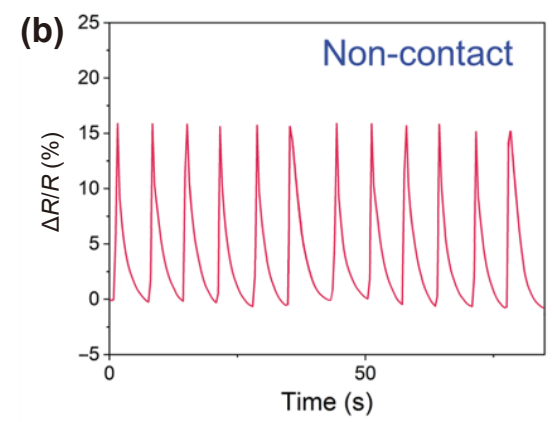

(c)

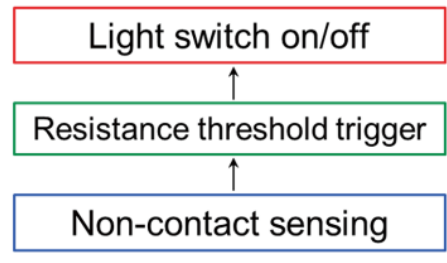

Circuit system logic

(d)

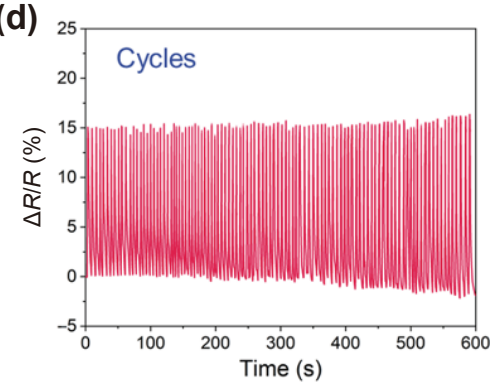

(g)

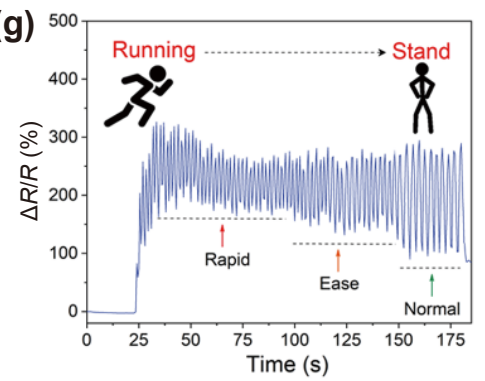

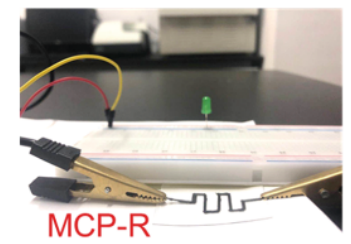
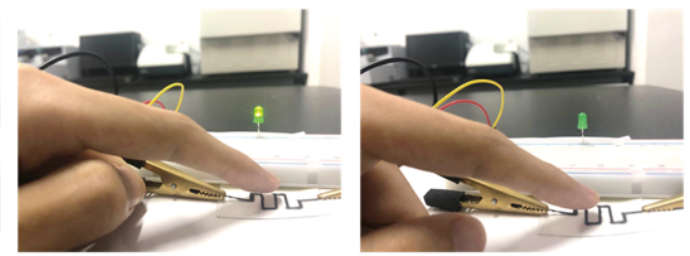

Non-contact sensing switch the light on-off
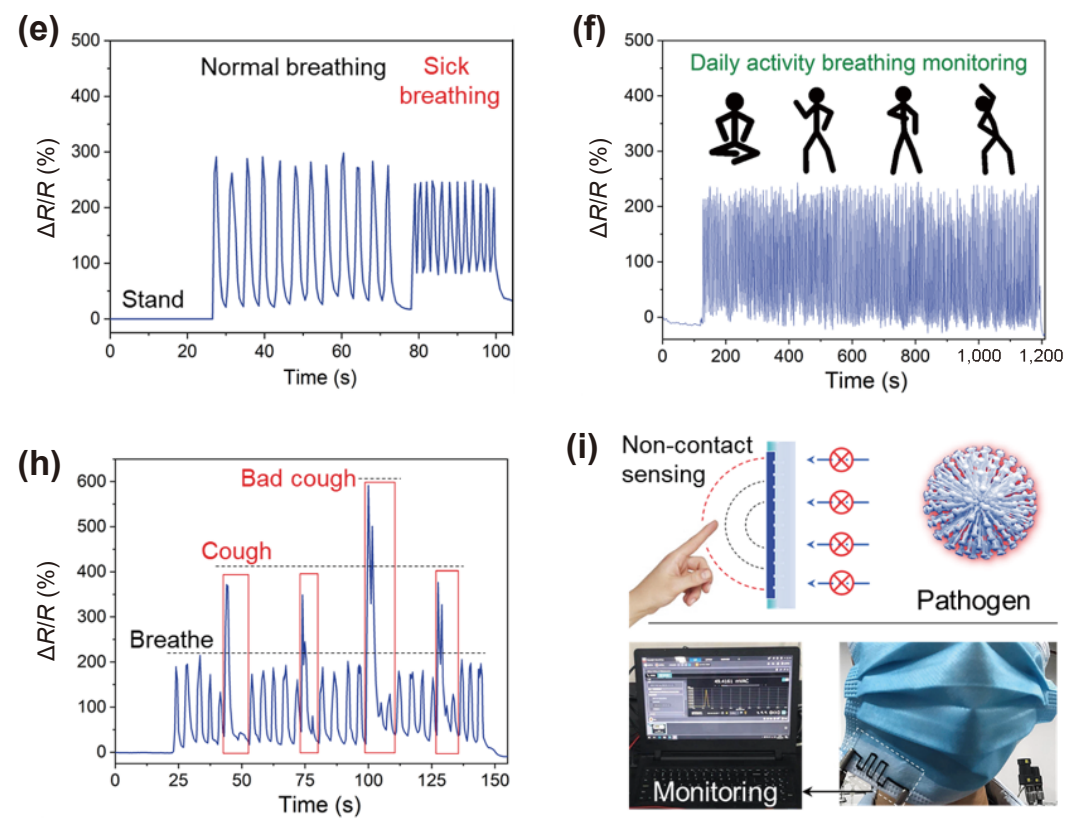

Figure 4 Non-contact sensing for block pathogens and warning of pneumonia symptoms by MCP E-skin. (a) Schematic diagram of non-contact sensing and finite element simulation. (b) Resistance response of non-contact sensing. (c) Demonstration of using MCP-R (R stands for resistance response mode) E-skin non-contact sensing capability to control LED light switches. (d) Resistance response of non-contact circulating test. (e) Resistance response of breathing. (f) Resistance response of daily activity breathing monitoring. (g) Resistance response of a man's breathing after running one kilometer. (h) Resistance response of the difference between breathing and coughing. (i) MCP E-skin blocks the contact infection of the pathogens through non-contact sensing, and respiratory monitoring to diagnose and treat diseases.

As shown in Fig. 4(h), the change in resistance response of cough was significantly greater than that of breathing. This is because coughing is the rapid elimination of air from the lungs and the more severe the cough, the more air will be exhausted in a short time. The mechanism of breathing and coughing response is that the MCP E-skin is sensitive to water vapor and carbon dioxide in the exhaled air, as shown in Fig. S11 in the ESM [47, 49]. Coughing may bring out a lot of water mist and cause the relaxation and recovery time of MCP E-skin to be longer, but the frequency of coughing is generally not too high. The MCP E-skin successfully captures breathing and cough signals, and can distinguish breathing conditions and cough frequency. These original signals can be amplified and rectified by the electrical system to obtain diagnostic signals (Fig. S12 in the ESM). We preliminary definition of cough as $500 \mathrm{~mL}$ of gas exhaled in $0.8 \mathrm{~s}$ and bad cough as $500 \mathrm{~mL}$ of gas exhaled in 0.5 s. Our measurements show that humans exhale less frequently during normal breathing or running, and generally exhale $500 \mathrm{~mL}$ of gas in more than $0.8 \mathrm{~s}$. These functions could be used in the monitoring of pneumonia patients and the early warning of severe pneumonia development. In short, as shown in Fig. 4(i), the noncontact sensing capability of the MCP electronic skin can effectively block the contact infection of the pathogen and it can also monitor the exercise monitoring and respiratory diagnosis, which is a very urgent application at the moment. MWCNT can be replaced by other promising nanomaterials to prepare materials with different functions, such as a nickel-chromium alloy flexible electric heating device in Fig. S13 in the ESM. The non-contact sensing capability of the MCP E-skin was endowed with high expectations to block pathogens from contacting infection. 
Compared with the E-skin with a single function and a commercial humidity sensor (HIH-5031-001, Honeywell), the multi-mode breath sensing function and higher stability brought more advantages to the MCP E-skin [46-48].

The electrostatic induction model was used to simulate in detail the resistance change when the finger approached the MCP Eskin. Approach the MCP E-skin slowly in the direction of the red arrow in Fig. 5(a) and record the resistance change curve for multiple tests, and then obtain Fig. 5(b). It shows that the resistance gradually increased when the finger slowly approached the MCP E-skin from $20 \mathrm{~mm}$ away, until there was a sine wave when touching. Among them, the purple area is the error bar, and the other test curves are shown in Fig. S14 in the ESM. The simulation curve in Fig. 5(c) was consistent with the test curve, which proved that the non-contact sensing mechanism of MCP Eskin was that the weak electrostatic field induction of the finger electrostatic field affected the electric field of the induction module, which illustrates the advanced performance of MCP Eskin. The finite element simulation of the finger approaching process the MCP E-skin is shown in Fig. 5(d). In order to verify the non-contact electronic control capability of the MCP E-skin, we selected a public light switch as a control to conduct a bacterial transfer experiment. Many bacteria have been isolated from public light switches, as shown in Fig. 5(e). The number and types of bacteria were surprising. We have isolated E. coli, S. aureus, and Bifidobacterium, which are pathogens that cause many kinds of inflammation in the human body [17-19]. Even more dangerous was that after the finger touched the light switch 40 times, a variety of bacteria and microorganisms were isolated from the finger (Fig. 5(e)). In contrast, by using the MCP electronic skin to noncontact switch the light 40-100 times, only a few oils and singledigit bacteria were isolated from the fingers (Fig. 5(f)). The bacteria transfer efficiency of the MCP electronic skin was only $1.36 \%$, while the public light switch was as high as $36.65 \%$. This shows that MCP electronic skin can effectively block the contact infection of public pathogens (Fig. 5(g)). The bacterial removal efficiency of MCP was tested using a similar method to that of Cui et al. [50]. From the results (Fig. 5(h)), it appeared that even if the MCP was accidentally contaminated with bacteria, it can be quickly sterilized by UV light irradiation.

\subsection{Tactile mapping of MCP E-skin}

Doctors and nurses in hospitals and isolation wards need to wear thick protective gloves during working hours, which affects their use of touch devices. We used the sine wave fluctuation at the moment of contact found in the non-contact sensing test (Fig. 5(b)) to fabricate an MCP-V (V stands for voltage response mode) that can achieve tactile response through contact (a)

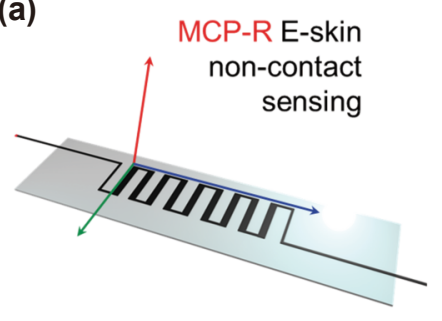

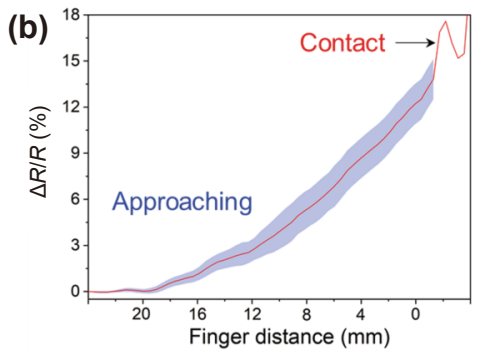

(c)

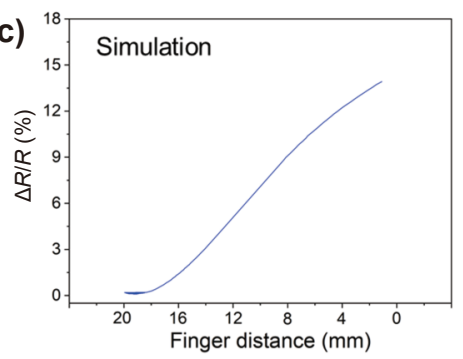

(d)

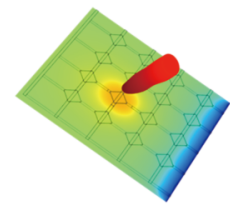

$d=20 \mathrm{~mm}$
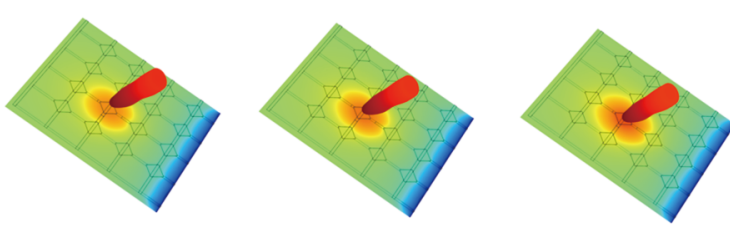

Finger distance from MCP-R

(e) Bacteria isolated from public facilities
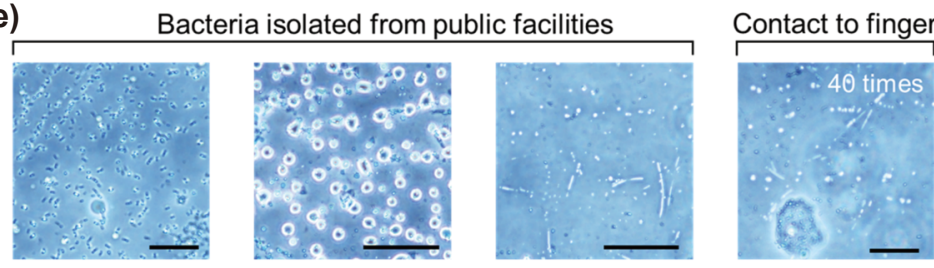

(f) No bacteria on the finger after using the non-contact sensing MCP E-skin
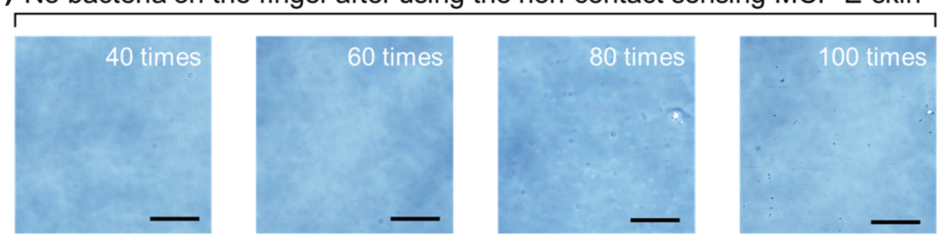

(h)

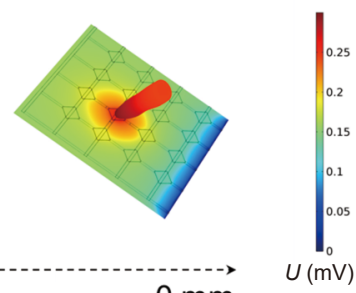

$0 \mathrm{~mm}$

(g)
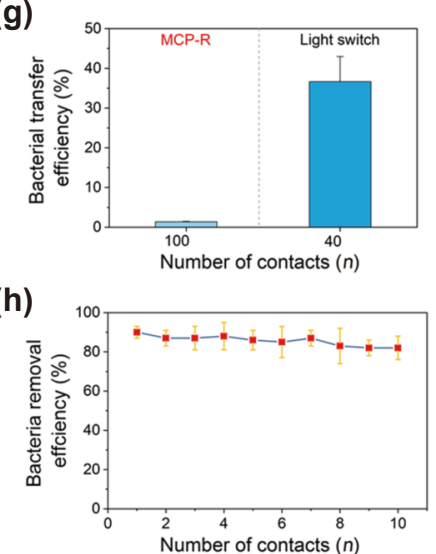

Figure 5 Non-contact sensing test and finite element simulation verification. (a) MCP E-skin resistance response (MCP-R) can achieve non-contact sensing. ((b) and (c)) Finger non-contact MCP-R resistance change test and finite element simulation data. (d) The finite element model simulates the change process of the electric potential field (potential difference, $U$ ) from $20 \mathrm{~mm}$ to the contact. (e) Microscope photos of E. coli, S. aureus, and Bifidobacterium isolated from the public light switch, as well as the bacteria transferred to finger after touching the public light switch 40 times. (f) Microscope photos of the bacteria transferred to finger after switching a LED by the MCP E-skin non-contact sensing 40, 60, 80, and 100 times. (g) Bacterial transfer efficiency of MCP E-skin and public light switch. Scale bar: $50 \mu \mathrm{m}$. (h) Bacterial removal efficiency of MCP E-skin. 
electrification. The protective gloves will also be charged with static electricity to cause contact electrification (Fig. 6(a)), and experimental tests have proved this process (Fig. 6(b)). Figure 6(c) shows a simple simulation scenario where the LED light was switched while wearing gloves. Through a circuit board connection, when the gloved finger touched the MCP E-skin for the first time, the voltage threshold was triggered, and the circuit automatically turned on the LED light; when the gloved finger touched again, the LED light off instruction was triggered. In addition, during this operation, the switch can be controlled by fingers with or without gloves. MCP E-skin can not only be used as a switch component in a single module, but also can be arranged in a matrix to achieve tactile mapping. Figure $6(\mathrm{~d})$ shows a $4 \times 4$ tactile sensor array and its tactile mapping. As shown in Fig. 6(e), the signal conversion circuit board had the functions of multi-channel collection of voltage signals, amplification, and rectification. The voltage signal generated by contact electrification is shown in the voltage distribution diagram on the right (Fig. 6(f)), and then the tactile mapping was synthesized. For the test video, see Video S3 in the ESM. The voltage response of MCP Eskin can quickly locate touch points, and can be able to monitor multi-touch and long touch. The software interface of the original data and the logic diagram of the circuit board are shown in Figs. S15 and S16 in the ESM. The rapid prototyping of the MCP Eskin implanted with MWCNT has proved its potential as a tactile sensor.

\section{Conclusions}

This research proposed a simple and effective implantation strategy to manufacture MCP E-skin, which can overcome multiple technical obstacles to adaptively fabricate E-skin on a flexible substrate. The mechanical interlocking structure of MWCNT/PVA gave MCP E-skin unique anti-damage property. The most fascinating non-contact sensing allowed it to be used as non-contact human-machine interfaces in public facilities to block the contact infection of pathogens. Respiration and cough sensing can be used for early warning and condition monitoring of exercise monitoring and respiratory diagnosis, which can be achieved by simply sticking the MCP E-skin on the mask. The new physical effects and structural design were applied to the MCP E-skin. At the same time, the MPC E-skin has been verified (a)

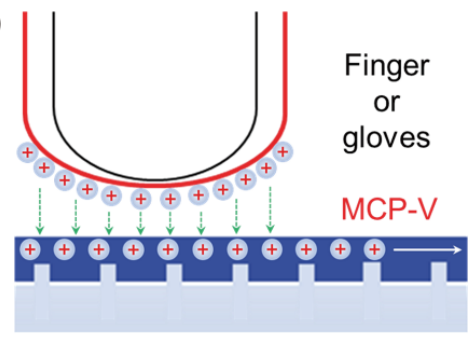

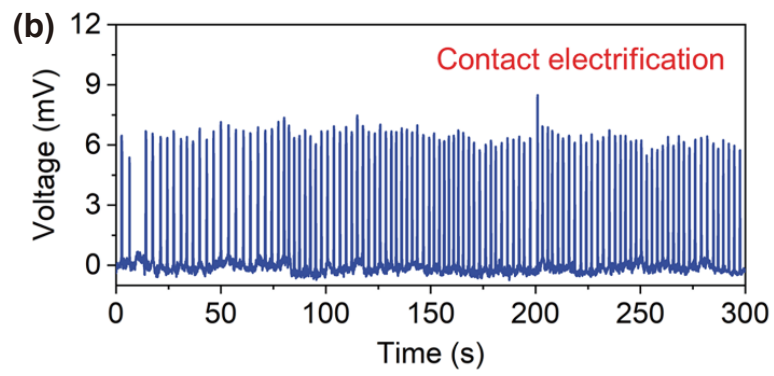

(c)

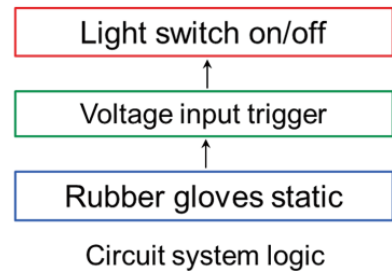

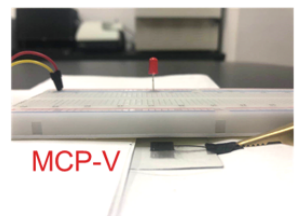

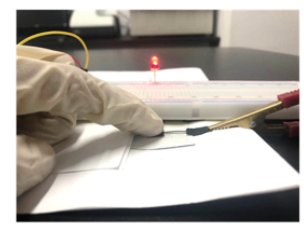

Finger wearing glove switch the light on-off (d)

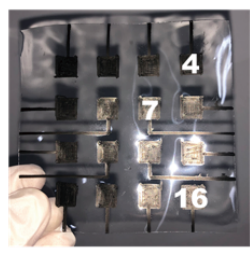

MCP-V

(e)

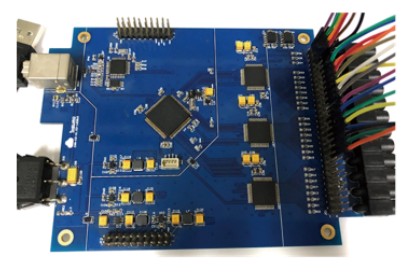

Signal conversion circuit board

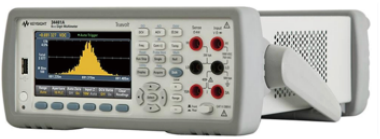

Tactile signal collection
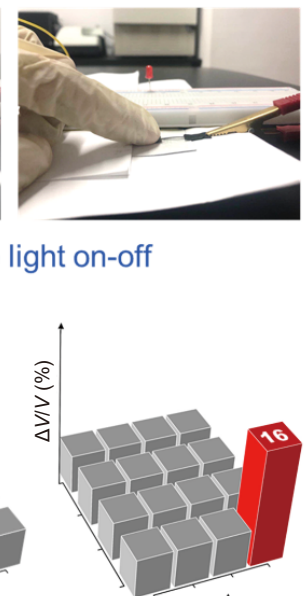

Tactile mapping
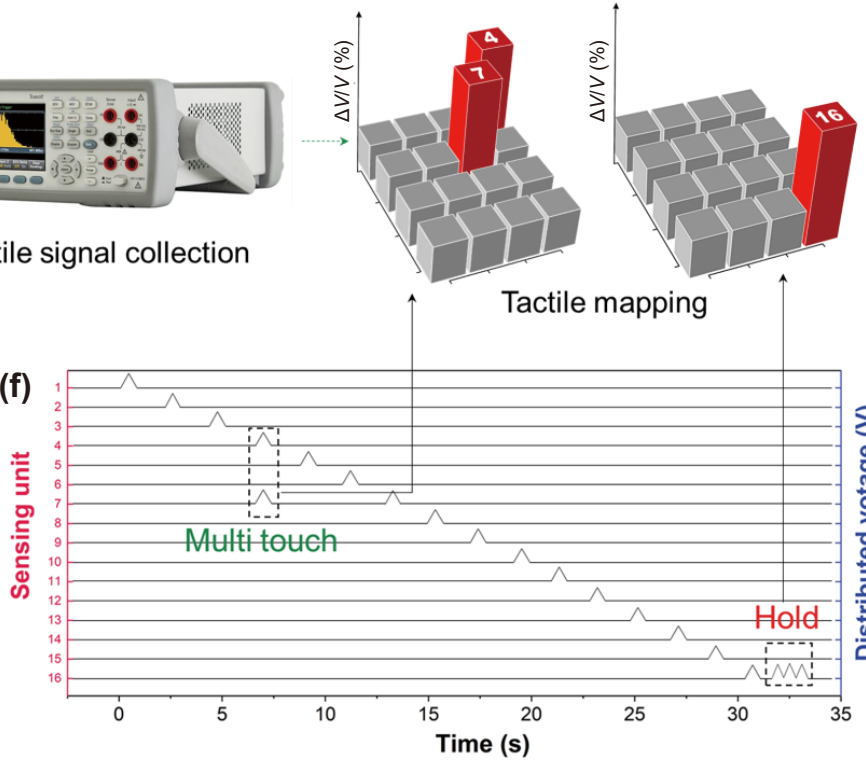

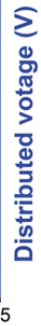

Figure 6 Demonstration of LED lights and tactile mapping for contact electrified switches. ((a) and (b)) Schematic diagram of finger or gloves electrostatic contact electrification with MCP E-skin and its open circuit voltage test. (c) Demonstration of using MCP E-skin contact electrification capability to switch LED light when wearing protective gloves. (d) A photo of MCP-V (V stands for voltage response mode) E-skin and the conversion process from tactile signal to tactile mapping. (e) A sensing module board of a multi-channel tactile detection system. (f) The voltage distribution signals of 16 touch points collected by the sensing module board. 
to be able to realize tactile mapping as a tactile sensor. Therefore, this research MCP E-skin can be applied not only in human-machine interaction with non-contact sensing, but also in the fields of gas sensors, wearable devices, soft electronics and intelligent robots.

\section{Experimental section}

\subsection{Fabrication of MCP E-skin}

PVA (molecular weight $\left(M_{\mathrm{W}}\right) \sim 6,700$, Shanghai Aladdin Biochemical Technology Co., Ltd.) membranes were prepared using the natural drying method. A PVA solution with a concentration of $10 \mathrm{wt} . \%$ was prepared by adding water and PVA to the flask and placing it in a water bath at $90{ }^{\circ} \mathrm{C}$. The PVA solution was then transferred to a square Petri dish for natural evaporation for $72 \mathrm{~h}$. The total PVA solution amount used determined the thickness of the final PVA film, and the shape of the square petri dish determined the size of the PVA film. The solutions for implanting were mixed liquid of MWCNT (Shanghai Aladdin Biochemical Technology Co., Ltd.) and commercial carbon ink (AICW9001, Shanghai M\&G Stationery INC.), and the solid-liquid mixing ratio was adjusted to explore the best process parameters. The tools for implanting were to cut off art fountain pens, and the head had both micro-engraving and diversion capabilities. The MCP E-skins were depicted by hand and an automatic $X-Y$ plotter (MCU assembled). The circuits of MCP Eskins were all written directly on the substrate, including PVA films, holly leaves, textile fabrics, and cellulose films. The tensile properties of PVA film and MCP were measured using a universal material testing machine (5943, Instron, UK) at a tensile rate of 10 $\mathrm{mm} / \mathrm{s}$. The contact angle was tested by a droplet shape analyzer (DSA100E, KRUSS, Germany).

\section{Acknowledgements}

The work was supported by Zhejiang Provincial Natural Science Key Foundation of China (No. LZ20E030003), National Science Foundation of China (No. 51673121), Candidates of Young and Middle Aged Academic Leader of Zhejiang Province, the Young Elite Scientists Sponsorship Program by CAST (No. 2018QNRC001), and Excellent Doctoral Thesis Cultivation Fund (No. 2019D01).

Electronic Supplementary Material: Supplementary material (fabrication of MCP E-skin, laser confocal tomography, parameter optimization, mechanical property characterization, finite element simulation, sensing mechanism, signal processing) is available in the online version of this article at https://doi.org/10.1007/s12274021-3831-z.

\section{References}

[1] Lee, G. H.; Moon, H.; Kim, H.; Lee, G. H.; Kwon, W.; Yoo, S.; Myung, D.; Yun, S. H.; Bao, Z. N.; Hahn, S. K. Multifunctional materials for implantable and wearable photonic healthcare devices. Nat. Rev. Mater. 2020, 5, 149-165.

[2] Liu, Y. Q.; He, K.; Chen, G.; Leow, W. R.; Chen, X. D. Natureinspired structural materials for flexible electronic devices. Chem. Rev. 2017, 117, 12893-12941.

[3] Xu, S. Y.; Vogt, D. M.; Hsu, W. H.; Osborne, J.; Walsh, T.; Foster, J. R.; Sullivan, S. K.; Smith, V. C.; Rousing, A. W.; Goldfield, E. C. et al. Biocompatible soft fluidic strain and force sensors for wearable devices. Adv. Funct. Mater. 2019, 29, 1807058.

[4] Wang, C. F.; Pan, C. F.; Wang, Z. L. Electronic skin for closed-loop systems. ACS Nano 2019, 13, 12287-12293.

[5] Choi, S.; Han, S. I.; Jung, D.; Hwang, H. J.; Lim, C.; Bae, S.; Park,
O. K.; Tschabrunn, C. M.; Lee, M.; Bae, S. Y. et al. Highly conductive, stretchable and biocompatible $\mathrm{Ag}-\mathrm{Au}$ core-sheath nanowire composite for wearable and implantable bioelectronics. Nat. Nanotechnol. 2018, 13, 1048-1056.

[6] Niu, S. M.; Matsuhisa, N.; Beker, L.; Li, J. X.; Wang, S. H.; Wang, J. C.; Jiang, Y. W.; Yan, X. Z.; Yun, Y. J.; Burnett, W. et al. A wireless body area sensor network based on stretchable passive tags. Nat. Electron. 2019, 2, 361-368.

[7] Wang, C. F.; Wang, C. H.; Huang, Z. L.; Xu, S. Materials and structures toward soft electronics. Adv. Mater. 2018, 30, 1801368.

[8] Kang, J. H.; Tok, J. B. H.; Bao, Z. N. Self-healing soft electronics. Nat. Electron. 2019, 2, 144-150.

[9] Yu, Y.; Nassar, J.; Xu, C. H.; Min, J. H.; Yang, Y. R.; Dai, A.; Doshi, R.; Huang, A.; Song, Y.; Gehlhar, R. et al. Biofuel-powered soft electronic skin with multiplexed and wireless sensing for humanmachine interfaces. Sci. Robot. 2020, 5, eaaz7946.

[10] Chortos, A.; Liu, J.; Bao, Z. N. Pursuing prosthetic electronic skin. Nat. Mater. 2016, 15, 937-950.

[11] Leber, A.; Dong, C. Q.; Chandran, R.; Das Gupta, T.; Bartolomei, N.; Sorin, F. Soft and stretchable liquid metal transmission lines as distributed probes of multimodal deformations. Nat. Electron. 2020, 3, 316-326.

[12] Markvicka, E. J.; Bartlett, M. D.; Huang, X. N; Majidi, C. An autonomously electrically self-healing liquid metal-elastomer composite for robust soft-matter robotics and electronics. Nat. Mater. 2018, 17, 618-624.

[13] Boutry, C. M.; Negre, M.; Jorda, M.; Vardoulis, O.; Chortos, A.; Khatib, O.; Bao, Z. N. A hierarchically patterned, bioinspired e-skin able to detect the direction of applied pressure for robotics. Sci. Robot. 2018, 3, eaau6914.

[14] Ong, S. W. X.; Tan, Y. K.; Chia, P. Y.; Lee, T. H.; Ng, O. T.; Wong, M. S. Y.; Marimuthu, K. Air, surface environmental, and personal protective equipment contamination by severe acute respiratory syndrome coronavirus 2 (SARS-CoV-2) from a symptomatic patient. JAMA 2020, 323, 1610-1612.

[15] Han, Y.; Yang, H. L. The transmission and diagnosis of 2019 novel coronavirus infection disease (COVID-19): A Chinese perspective. J. Med. Virol. 2020, 92, 639-644.

[16] Kaper, J. B.; Nataro, J. P.; Mobley, H. L. T. Pathogenic Escherichia coli. Nat. Rev. Microbiol. 2004, 2, 123-140.

[17] Kretschmer, D.; Gleske, A. K.; Rautenberg, M.; Wang, R.; Köberle, M.; Bohn, E.; Schöneberg, T.; Rabiet, M. J.; Boulay, F.; Klebanoff, S. J. et al. Human formyl peptide receptor 2 senses highly pathogenic Staphylococcus aureus. Cell Host Microbe 2010, 7, 463-473.

[18] Hay, R.; Bendeck, S. E.; Chen, S.; Estrada, R.; Haddix, A.; McLeod, T.; Mahé, A. Skin diseases. In Disease Control Priorities in Developing Countries. Jamison, D. T.; Breman, J. G., Eds.; World Bank Publications: Washington DC, 2006, pp 707-722.

[19] Malm, H.; Navin, M. C. Pox parties for grannies? Chickenpox, exogenous boosting, and harmful injustices. Am. J. Bioeth. 2020, 20, $45-57$.

[20] Hoang, C. Q.; Nguyen, T. T. T.; Ho, N. X.; Nguyen, H. D.; Nguyen, A. B.; Nguyen, T. H. T.; Phan, H. C.; Phan, L. T. Transmission and serotype features of hand foot mouth disease in household contacts in Dong Thap, Vietnam. BMC Infect. Dis. 2019, 19, 933.

[21] Mina, M. J.; Kula, T.; Leng, Y. M.; Li, M. M.; de Vries, R. D.; Knip, M.; Siljander, H.; Rewers, M.; Choy, D. F.; Wilson, M. S. et al. Measles virus infection diminishes preexisting antibodies that offer protection from other pathogens. Science 2019, 366, 599-606.

[22] Sun, H. L.; Zhao, Y.; Wang, C. F.; Zhou, K. K.; Yan, C.; Zheng, G. Q.; Huang, J. J.; Dai, K.; Liu, C. T.; Shen, C. Y. Ultra-stretchable, durable and conductive hydrogel with hybrid double network as high performance strain sensor and stretchable triboelectric nanogenerator. Nano Energy 2020, 76, 105035.

[23] Ye, T. L.; Wang, Q.; Tian, C. H.; Singh, R.; Zhang, Y. P.; Liu, Z. W.; Fang, X K.; He, D. Q. Multifunctional electronic skin based on perovskite intermediate gels. Adv. Electron. Mater. 2020, 6, 1901291.

[24] Zhao, D. W.; Zhu, Y.; Cheng, W. K.; Xu, G. W.; Wang, Q. W.; Liu, 
S. X.; Li, J.; Chen, C. J.; Yu, H. P.; Hu, L. B. A dynamic gel with reversible and tunable topological networks and performances. Matter 2020, 2, 390-403.

[25] Qiao, Y. C.; Wang, Y. F.; Tian, H.; Li, M. R.; Jian, J. M.; Wei, Y. H.; Tian, Y.; Wang, D. Y.; Pang, Y.; Geng, X. S. et al. Multilayer graphene epidermal electronic skin. ACS Nano 2018, 12, 8839-8846.

[26] Won, S. M.; Wang, H. L.; Kim, B. H.; Lee, K.; Jang, H.; Kwon, K.; Han, M. D.; Crawford, K. E.; Li, H. B.; Lee, Y. et al. Multimodal sensing with a three-dimensional piezoresistive structure. ACS Nano 2019, 13, 10972-10979.

[27] Mu, C. H.; Song, Y. Q.; Huang, W. T.; Ran, A.; Sun, R. J.; Xie, W. H.; Zhang, H. W. Flexible normal-tangential force sensor with opposite resistance responding for highly sensitive artificial skin. Adv. Funct. Mater. 2018, 28, 1707503.

[28] Hong, S. Y.; Lee, Y. H.; Park, H.; Jin, S. W.; Jeong, Y. R.; Yun, J.; You, I.; Zi, G.; Ha, J. S. Stretchable active matrix temperature sensor array of polyaniline nanofibers for electronic skin. Adv. Mater. 2016, 28, 930-935.

[29] Yang, J. C.; Mun, J.; Kwon, S. Y.; Park, S.; Bao, Z. N.; Park, S. Electronic skin: Recent progress and future prospects for skinattachable devices for health monitoring, robotics, and prosthetics. Adv. Mater. 2019, 31, 1904765.

[30] Wu, Y. H.; Deng, Z. F.; Peng, Z. F.; Zheng, R. M.; Liu, S. Q.; Xing, S. T.; Li, J. Y.; Huang, D. Q.; Liu, L. A novel strategy for preparing stretchable and reliable biphasic liquid metal. Adv. Funct. Mater. 2019, 29, 1903840.

[31] Tang, Y. J.; Zhou, H.; Sun, X. P.; Diao, N. H.; Wang, J. B.; Zhang, B. S.; Qin, C.; Liang, E. J.; Mao, Y. C. Triboelectric touch-free screen sensor for noncontact gesture recognizing. Adv. Funct. Mater. 2020, 30, 1907893.

[32] Kang, M.; Kim, J.; Jang, B.; Chae, Y.; Kim, J. H.; Ahn, J. H. Graphene-based three-dimensional capacitive touch sensor for wearable electronics. ACS Nano 2017, 11, 7950-7957.

[33] Zhao, J.; Li, N.; Yu, H.; Wei, Z.; Liao, M. Z.; Chen, P.; Wang, S. P.; Shi, D. X.; Sun, Q. J.; Zhang, G. Y. Highly sensitive $\mathrm{MoS}_{2}$ humidity sensors array for noncontact sensation. Adv. Mater. 2017, 29, 1702076.

[34] Feng, J.; Peng, L. L.; Wu, C. Z.; Sun, X.; Hu, S. L.; Lin, C. W.; Dai, J.; Yang, J. L.; Xie, Y. Giant moisture responsiveness of $\mathrm{VS}_{2}$ ultrathin nanosheets for novel touchless positioning interface. $A d v$. Mater. 2012, 24, 1969-1974.

[35] Jiao, J. P.; Li, L.; Wu, B.; He, C. F. Novel capacitive proximity sensors for assessing the aging of composite insulators. Sens. Actuators A Phys. 2017, 253, 75-84.

[36] Thostenson, E. T.; Ren, Z. F.; Chou, T. W. Advances in the science and technology of carbon nanotubes and their composites: A review. Compos. Sci. Technol. 2001, 61, 1899-1912.

[37] Dasbach, M.; Pyschik, M.; Lehmann, V.; Parey, K.; Rhinow, D.; Reinhardt, H. M.; Hampp, N. A. Assembling carbon nanotube architectures. ACS Nano 2020, 14, 8181-8190.

[38] Halima, N. B. Poly (vinyl alcohol): Review of its promising applications and insights into biodegradation. RSC Adv. 2016, 6, 39823-39832.

[39] Peng, X.; Dong, K.; Ye, C. Y.; Jiang, Y.; Zhai, S. Y.; Cheng, R. W.; Liu, D.; Gao, X. P.; Wang, J.; Wang, Z. L. A breathable, biodegradable, antibacterial, and self-powered electronic skin based on all-nanofiber triboelectric nanogenerators. Sci. $A d v$. 2020, 6, eaba9624.

[40] Wang, Y.; Zhang, L. N.; Lu, A. Highly stretchable, transparent cellulose/PVA composite hydrogel for multiple sensing and triboelectric nanogenerators. J. Mater. Chem. A 2020, 8, 13935-13941.

[41] Wang, X. D.; Zhang, Y. F.; Zhang, X. J.; Huo, Z. H.; Li, X. Y.; Que, M. L.; Peng, Z. C.; Wang, H.; Pan, C. F. A highly stretchable transparent self-powered triboelectric tactile sensor with metallized nanofibers for wearable electronics. Adv. Mater. 2018, 30, 1706738.

[42] Pizzi, A.; Mittal, K. L. Handbook of Adhesive Technology; 3rd ed. CRC Press: Boca Raton, 2017.

[43] Rance, G. A.; Marsh, D. H.; Nicholas, R. J.; Khlobystov, A. N. UVvis absorption spectroscopy of carbon nanotubes: Relationship between the $\pi$-electron plasmon and nanotube diameter. Chem. Phys. Lett. 2010, 493, 19-23.

[44] Kaiser, A. B.; Flanagan, G. U.; Stewart, D. M.; Beaglehole, D. Heterogeneous model for conduction in conducting polymers and carbon nanotubes. Synth. Met. 2001, 117, 67-73.

[45] Kaiser, A. B. Thermoelectric power and conductivity of heterogeneous conducting polymers. Phys. Rev. B 1989, 40, 2806-2813.

[46] Li, Z. D.; Liu, H.; Ouyang, C.; Wee, W. H.; Cui, X. Y.; Lu, T. J.; Pingguan - Murphy, B.; Li, F.; Xu, F. Recent advances in pen-based writing electronics and their emerging applications. Adv. Funct. Mater. 2016, 26, 165-180.

[47] Wang, W. Y.; Ouaras, K.; Rutz, A. L.; Li, X.; Gerigk, M.; Naegele, T. E.; Malliaras, G. G.; Huang, Y. Y. S. Inflight fiber printing toward array and $3 \mathrm{D}$ optoelectronic and sensing architectures. Sci. Adv. 2020, 6, eaba0931.

[48] Shin, J.; Jeong, B.; Kim, J.; Nam, V. B.; Yoon, Y.; Jung, J.; Hong, S.; Lee, H.; Eom, H.; Yeo, J. et al. Sensitive wearable temperature sensor with seamless monolithic integration. Adv. Mater. 2020, 32, 1905527.

[49] Rahimabady, M.; Tan, C. Y.; Tan, S. Y.; Chen, S.; Zhang, L.; Chen, Y. F.; Yao, K.; Zang, K. Y.; Humbert, A.; Soccol, D. et al. Dielectric nanocomposite of diphenylethylenediamine and P-type multi-walled carbon nanotube for capacitive carbon dioxide sensors. Sens. Actuators B Chem. 2017, 243, 596-601.

[50] Liao, L.; Xiao, W.; Zhao, M.; Yu, X. Z.; Wang, H. T.; Wang, Q. Q.; Chu, S.; Cui, Y. Can N95 respirators be reused after disinfection? How many times? ACS Nano 2020, 14, 6348-6356. 